\title{
OBSERVED VARIABILITY OF THE SOLAR Mg II h SPECTRAL LINE
}

\author{
D. Schmit ${ }^{1,2}$, P. Bryans ${ }^{3}$, B. De Pontieu ${ }^{1}$, S. McIntosh ${ }^{3}$, J. Leenaarts ${ }^{4}$, and M. Carlsson ${ }^{5}$ \\ ${ }^{1}$ Lockheed-Martin Solar and Astrophysics Laboratory, Palo Alto, CA, USA \\ ${ }^{2}$ Bay Area Environmental Research Institute, Petaluma, CA, USA \\ ${ }^{3}$ High Altitude Observatory, National Center for Atmospheric Research, Boulder, CO, USA \\ ${ }^{4}$ Institutet för solfysik, Stockholms Universitet, Stockholm, Sweden \\ ${ }^{5}$ Institute of Theoretical Astrophysics, University of Oslo, Oslo, Norway \\ Received 2015 June 2; accepted 2015 August 17; published 2015 September 29
}

\begin{abstract}
The $\mathrm{Mg}$ II $\mathrm{h} \& \mathrm{k}$ doublet are two of the primary spectral lines observed by the Sun-pointing Interface Region Imaging Spectrograph (IRIS). These lines are tracers of the magnetic and thermal environment that spans from the photosphere to the upper chromosphere. We use a double-Gaussian model to fit the $\mathrm{Mg}$ II $\mathrm{h}$ profile for a full-Sun mosaic data set taken on 2014 August 24 . We use the ensemble of high-quality profile fits to conduct a statistical study on the variability of the line profile as it relates the magnetic structure, dynamics, and center-to-limb viewing angle. The average internetwork profile contains a deeply reversed core and is weakly asymmetric at $\mathrm{h} 2$. In the internetwork, we find a strong correlation between $\mathrm{h} 3$ wavelength and profile asymmetry as well as $\mathrm{h} 1 \mathrm{width}$ and $\mathrm{h} 2$ width. The average reversal depth of the $\mathrm{h} 3$ core is inversely related to the magnetic field. Plage and sunspots exhibit many profiles that do not contain a reversal. These profiles also occur infrequently in the internetwork. We see indications of magnetically aligned structures in plage and network in statistics associated with the line core, but these structures are not clear or extended in the internetwork. The center-to-limb variations are compared to predictions of semi-empirical model atmospheres. We measure a pronounced limb darkening in the line core that is not predicted by the model. The aim of this work is to provide a comprehensive measurement baseline and preliminary analysis on the observed structure and formation of the $\mathrm{Mg}$ II profiles observed by IRIS.
\end{abstract}

Key words: Sun: chromosphere - Sun: UV radiation

\section{INTRODUCTION}

The magnetic structure and thermodynamics of the solar chromosphere are open problems in solar physics with broad implications for stellar atmospheres. The visible spectrum contains several lines $(\mathrm{H} \alpha, \mathrm{Ca}$ II $\mathrm{H} \& \mathrm{~K})$ that have allowed us to probe the structure and dynamics of the chromosphere using ground-based observatories. The recent launch of the Interface Region Imaging Spectrograph (IRIS; De Pontieu et al. 2014) provides a new data set observing one of the most important radiators of the chromosphere, the $\mathrm{Mg}$ II h\&k lines at $2803.5 \AA$ and $2796.4 \AA$, respectively. While there have been numerous missions to observe the $\mathrm{Mg}$ II $\mathrm{h} \& \mathrm{k}$ lines, the measurements have been sparse. The earliest measurements were done using a rocket-borne spectrograph (Durand et al. 1949). The first study to derive structural variations in the profiles was that of Lemaire \& Skumanich (1973). The OSO-8 (Artzner et al. 1977) and Skylab (Doschek \& Feldman 1977) missions provided the first orbital data sets. The Solar Maximum Mission made the first polarization measurements of the $\mathrm{Mg}$ line profiles (Henze \& Stenflo 1987). The highest spectral resolution measurements of the profile prior to IRIS were made by the HRTS rocket (Morrill et al. 2001) and the RASOLBA balloon (Staath \& Lemaire 1995) spectrographs. Center-to-limb measurements were discussed by Gouttebroze \& Lemaire (1974), Bonnet (1981), and Morrill \& Korendyke (2008). These measurements have been used to construct model atmospheres for a variety of solar structures. Gouttebroze $(1977,1989)$ modeled the effect of velocity gradients in the internetwork chromosphere on profile asymmetry. Lemaire et al. (1981) synthesized profiles for plage. Umbral profiles, which were originally identified as uniquely single peaked, have been studied by Kneer et al. (1981), Lites \& Skumanich (1982), and Gurman (1984). Limb observations in $\mathrm{Mg}$ II also provided diagnostics of prominences (Vial et al. 1979).

In addition to the data points provided by solar observations, multiple missions have conducted studies of stellar emission in the $\mathrm{Mg}_{\text {II }}$ lines. The shape of $\mathrm{Mg}$ II $\mathrm{h} \& \mathrm{k}$ varies significantly across stellar types: from pure absorption in Altair to singlepeaked emission in $\epsilon$ Eri (Basri \& Linsky 1979; Blanco et al. 1982). The differences between the shape of $M g$ II $h \& k$ are a strong diagnostic of stellar winds and shocks in extended chromospheres like that of $\alpha$ Ori (Uitenbroek et al. 1998). Variability and activity cycles have been detected in the $\mathrm{Mg}$ II h\&k lines in other stars (Dempsey et al. 1996). While stellar chromospheres exist in some form across the cooler half of the main sequence, there is a complicated relationship with coronae: a chromosphere is a prerequisite to form a corona, but stars with a chromosphere do not need to have a corona (Linsky \& Haisch 1979). The chromosphere-corona link is an important one for solar-terrestrial studies. Solar irradiance is a driving factor in determining the ion populations of the Earth's upper atmosphere (Solomon \& Qian 2005). While Mg II h\&k are unlikely to play a significant role in thermospheric photoionization, $\mathrm{Mg}$ II irradiance has been shown to be a superior proxy to EUV irradiance over the F10.7 radio index (Guo et al. 2007; Lean et al. 2009).

The complex profiles observed in the solar $\mathrm{Mg}$ II doublet can be attributed to the lines' complex formation. A seminal study was presented in Milkey \& Mihalas (1974). The Mg II doublet are resonance lines for a highly abundant element. As such, they are very optically thick at the line cores, which form at relatively low densities under NLTE conditions. The formation depth of the profile varies significantly with wavelength. The core is estimated to form between 1 and $3 \mathrm{Mm}$ above the $\left(\tau_{500 \mathrm{~nm}}=1\right)$ photosphere. At $\pm 0.5 \AA$, the line opacity is 
reduced such that the formation layer maps near the temperature minimum, $500 \mathrm{~km}$ above the photosphere. Leenaarts et al. (2013a, 2013b) and Pereira et al. (2013) used a radiative MHD numerical simulation (Gudiksen et al. 2011) to forward model the emission of the $\mathrm{Mg}$ II lines. These authors describe in detail the formation mechanisms of the lines and how line components can be transformed into practical diagnostics of the solar atmosphere. Our research is complementary to these papers. In this paper, we delve into the profiles of $\mathrm{Mg}$ II $\mathrm{h}$ observed by IRIS. We fit the line profile with a double-Gaussian model. A post-processing routine parses the best-fit model (BFM) into a variety of profile statistics. We analyze a full-Sun mosaic data set that offers us several million profiles, allowing us to present an overview of how the line components vary over the structurally distinct regions of the Sun. In particular, we analyze the variation of $\mathrm{Mg}$ II $\mathrm{h}$ with magnetic structure, dynamics, and viewing angle. Section 2 presents the observations and data reduction. Section 3 presents methodology for fitting the profiles and deriving the profile statistics. Section 4 describes the types of profiles observed and interpretation on the physical sources of variability. Section 5 is summarizes the results and discusses what future steps need to be taken.

\section{DATA}

IRIS is designed to investigate the heating of the solar atmosphere by collecting spectra and images of the Sun in three ultraviolet passbands: 1332-1358 $\AA$, 1381-1407 $\mathrm{\AA}$, and 2783-2834 A. IRIS obtains spectral information by passing light from the Sun through a slit and onto a grating. The spatial range of the spectra are limited by the 175 arcsec length of the slit. Temporal limitations are introduced by the exposure time needed to build up sufficient photon counts and by rastering the slit across the solar disk.

We have designed an observing sequence that uses the capabilities of IRIS to build up a spectral map of the entire solar disk over as short a time as is practical. To generate a full-Sun observation with the limited field of view of IRIS, we take successive observations at different satellite pointings and build up a mosaic of the Sun; 184 different observations were needed to generate the full-Sun mosaic. Each individual observation consists of a 64-step raster with 2 arcsec steps and $2 \mathrm{~s}$ exposure time at each slit position. The spectra along the slit have been binned to a resolution of 0.66 arcsec. This gives an area of $128 \times 175$ arcsec that takes $\sim 190 \mathrm{~s}$ to observe. All 184 positions take $\sim 18 \mathrm{hr}, \sim 10 \mathrm{hr}$ of which is observing time with the remainder in repointing the spacecraft.

The above observing sequence is currently run approximately once per month when IRIS is not in eclipse season. For the purposes of this study, we have chosen one such observation from 2014 August 24 12:16 UT to 2014 August 25 05:40 UT. The data used in this paper are IRIS level 2 data products. These data have been processed from the raw observations to remove bad pixels resulting from dust on the detector, dark current and flat-field corrections have been implemented, and geometric and wavelength calibrations (based on the rest wavelength of Ni I $2799.17 \AA$ ) applied. For a complete description of the calibration process, see the IRIS user guide. ${ }^{6}$ The full-Sun mosaic was assembled by positioning each raster according to its associated spacecraft pointing.

\footnotetext{
6 http://iris.lmsal.com/itn26/
}

There are two sections of the mosaic that were observed while the orbit of IRIS was affected by the South Atlantic Anomaly. There regions are seen at low latitude near the east and west limbs in Figure 2(a). Due to an increase in cosmic-ray hits at these times, we choose to remove these data from our analysis.

This observation contains spectral information for several wavelength windows within the IRIS NUV and FUV passbands. For the purposes of this study, however, we limit our analysis to the $\mathrm{Mg}$ II $\mathrm{h}$ line at $2803.5 \AA$. We analyze a spectral window $3.4 \AA$ wide, centered at the $\mathrm{Mg}$ II $\mathrm{h}$ line, with resolution of $\sim 52 \mathrm{~mA}$. In future work, we intend to expand this analysis to include other emission and absorption features of the IRIS mosaic data sets.

In order to compare the IRIS data with the photospheric magnetic field, we use simultaneous observations from the Helioseismic and Magnetic Imager (HMI; Scherrer et al. 2012) on board the Solar Dynamics Observatory (Pesnell et al. 2012). HMI provides the line of sight component of the magnetic field for the entire Earth-facing solar disk at a cadence of $720 \mathrm{~s}$. We combine many of these observations to arrive at a full-Sun line of sight (LOS) magnetic field map that most closely matches the times of observation of the IRIS data. For each IRIS raster, we select the HMI observation that was temporally closest, and then we select the portion of the HMI observation that matches the IRIS field of view. By repeating this process for all 184 IRIS rasters, we can construct an HMI image of the full Sun.

\section{METHOD}

We have chosen to use a nine-parameter double-Gaussian model to fit a $3.4 \AA$ wide window centered on $\mathrm{Mg}$ II h:

$$
\begin{aligned}
I(\lambda)= & a+b *|\lambda-c|+d *\left(\exp \left(\frac{-(\lambda-f)^{2}}{g^{2}}\right)\right. \\
& \left.-h * \exp \left(\frac{-(\lambda-j)^{2}}{k^{2}}\right)\right),
\end{aligned}
$$

where the units of $[I, a, d]$ are $\mathrm{DN}, b$ is $\mathrm{DN} \AA^{-1}$, and $[c, f, g, j, k]$ are $\AA$. Empirically, we find that this is an accurate model for a majority of the observed profiles. The $\mathrm{Mg}$ II $\mathrm{h}$ line is optically thick, and the formation height of the profile varies by hundreds of kilometers from h1 to h3. To calculate a theoretical profile, a synthetic atmosphere must be used to derive the source function as a function of wavelength along the line of sight. Our fit model does not attempt to include these physics; however, the the double-Gaussian model provides an easily parameterized model that is capable of producing both single- and double-peaked profiles with incongruous widths in the wing and core. The average solar $\mathrm{Mg}$ II h\&k profile is an emission line with a reversed (depressed) core, which is captured by the superposition of a wide positive amplitude Gaussian and a narrow negative amplitude Gaussian. The far wings of the profile are captured by the linear function. While this technique is applicable to $\mathrm{Mg}$ II $\mathrm{k}$ as well, that analysis is complicated by the presence of the Mn I line at $2795.6 \AA$.

We use the MPFIT least squares minimization algorithm (Markwardt 2009) to derive a BFM. We assume the measurement errors are the linear combination of Poisson noise (based on the 18 photon per DN estimate in De Pontieu et al. 2014) and a constant readout noise of 3 DN. After a BFM (in terms of $\chi^{2}$ ) is retrieved from the minimization routine, we 

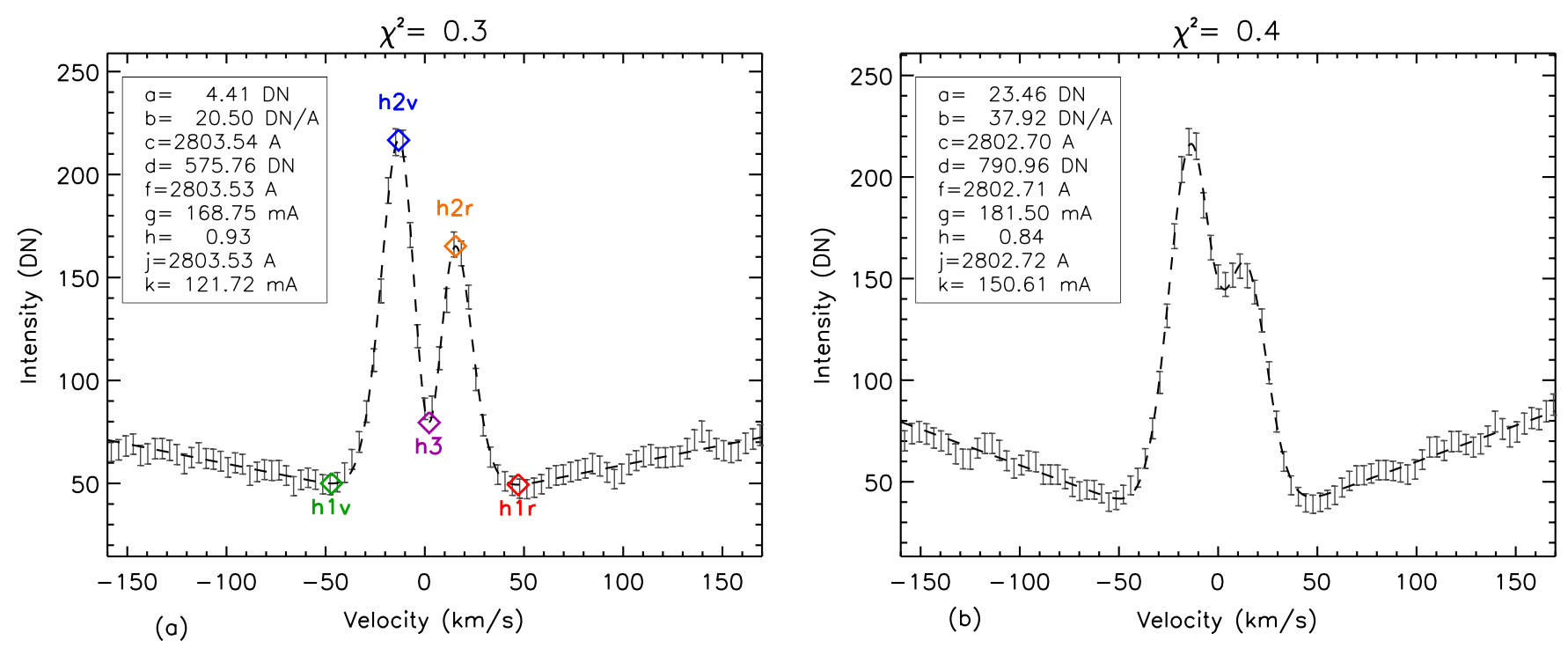

Figure 1. Typical double-peaked profiles in the internetwork. Gray error bars show the IRIS data. The best-fit model is plotted in dashed black. The BFM parameters for Equation (1) are listed in the legend.

apply an algorithm to determine the number and location of local extrema in the BFM. The average profile will have two maxima at the red and blue edge of the spectral window (see Figure 1; at $170 \mathrm{~km} \mathrm{~s}^{-1}$ and $-160 \mathrm{~km} \mathrm{~s}^{-1}$, respectively) that bound three minima (h1v, h3, h1r) and two additional maxima $(\mathrm{h} 2 \mathrm{v}, \mathrm{h} 2 \mathrm{r})$. The BFM value at these spectral positions is recorded along with the position. One additional case is accepted as a viable model: three maxima-two minima (single peak with weak wings). While some profiles are found with negative value for $b$ in Equation (1), these profiles are the result of noisy spectrums or high total $\chi^{2}$.

Incorporated with the IRIS package available for Solarsoft is the iris_get_mg_features algorithm that may also be used to identify the $\mathrm{h} 2$ peaks and $\mathrm{h} 3$ minimum (but not $\mathrm{h} 1$ minima) of the $\mathrm{h}$ line. We have opted for a fitting-based approach to derive a comprehensive parameterization of the profile that uses all the available data. A low- $\chi^{2}$ fit represents an accurate model, while a high- $\chi^{2}$ fit can be further examined to determine how the model has failed. In a test sample, our fitting method and iris_get_mg_features differed by less than a spectral pixel for $\lambda_{\mathrm{h} 3}$ for $85 \%$ of profiles.

We created structural masks to aid in parsing the data set. The internetwork and network subsets were identified using the $S D O / \mathrm{HMI}$ LOS magnetic field map. The magnetic qualifier for internetwork is less than $5 \%$ of the pixels $\left(0{ }^{\prime \prime} 5\right.$ pixels in the native resolution) within a 5 pixel radius could have an unsigned LOS strength greater than $60 \mathrm{G}$. For network, more than $15 \%$ of pixels need to exceed that magnetic threshold. Plage was identified as large contiguous regions of unsigned magnetic flux with high intensities at $2803.3 \AA$ (far enough from into the wing to be unaffected by the highly variable core intensity). The distinction between large network concentrations and plage was made manually based on the unipolarity of the nearby area. Sunspots were identified as contiguous regions of high unsigned magnetic flux with low intensities $(<40 \mathrm{DN})$ at $2801.8 \AA$ (the outer most extent of our spectral window and the closest to continuum intensity). Filaments were identified using only a spectral qualifier. Filaments were selected as contiguous regions where the line core intensity drops below $38 \mathrm{DN}$. Table 1 identifies the number of profiles in each region.
Table 1

Number of Profiles in Each Structural Region of the 2014 August 24 Data Set

\begin{tabular}{lr}
\hline \hline Structure & Profiles $\left(\chi^{2}<1.5\right)$ \\
\hline Internetwork & $3.8(3.3) \times 10^{6}$ \\
Network & $2.1(1.4) \times 10^{5}$ \\
Plage & $1.2(0.72) \times 10^{5}$ \\
Sunspot & $3.8(3.4) \times 10^{4}$ \\
Filament & $1.4(1.2) \times 10^{5}$ \\
\hline
\end{tabular}

Note. Profiles with a qualifying $\chi^{2}$ are listed in parentheses.

Figure 1 shows typical profiles observed by IRIS in the internetwork. The extrema of the fit are labeled in the standard method: the reversed core is $\mathrm{h} 3$, the emission peaks are $\mathrm{h} 2$, and minimum in the wing is $h 1, v$ and $r$ refer to the violet and red side of the profile. Our models fits return reduced $\chi^{2}$ values of 0.4 , which imply that our errors are a liberal estimate of the noise in the spectra. Approximately $48 \%$ of the on-disk spectra have fits with lower $\chi^{2}$ than these models. We use $\chi^{2}<1.5$ as our viable model threshold, which applies to $86 \%$ of our data set.

The profiles in Figures 1(a) and (b) have much in common and simultaneously have many differences. Both profiles are double peaked, but the two peaks are not identical in intensity. To measure this difference we use the asymmetry statistic:

$$
R_{\mathrm{h}}=\frac{I_{\mathrm{h} 2 \mathrm{v}}-I_{\mathrm{h} 2 \mathrm{r}}}{I_{\mathrm{h} 2 \mathrm{v}}+I_{\mathrm{h} 2 \mathrm{r}}}
$$

which is also used in Pereira et al. (2013). Figures 1(a) and (b) have $R_{\mathrm{h}}$ values of 0.13 and 0.15 , respectively. The h1 width $\left(\lambda_{\mathrm{h} 1 \mathrm{r}}-\lambda_{\mathrm{h} 1 \mathrm{v}}\right)$ and $\mathrm{h} 2$ width $\left(\lambda_{\mathrm{h} 2 \mathrm{r}}-\lambda_{\mathrm{h} 2 \mathrm{v}}\right)$ are also similar for these profiles. However, the core of the profiles are strikingly different. Both profiles have a h3 minimum, but Figure 1(a) has a much deeper core depression. We measure the relative depth of the core using the depth statistic:

$$
D_{\mathrm{h}}=1-\frac{2 I_{\mathrm{h} 3}}{I_{\mathrm{h} 2 \mathrm{v}}+I_{\mathrm{h} 2 \mathrm{r}}} \text {. }
$$

Figures 1(a) and (b) have a $D_{\mathrm{h}}$ of 0.58 and 0.23 , respectively. The differences of these profiles are emblematic of the 
complexity we observe in high-resolution IRIS $\mathrm{Mg}$ II spectra. The correlations found in Leenaarts et al. (2013b) suggest that the intensity at the $h 3$ core is inversely related to the altitude of the $\tau=1$ layer, implying that Figure 1(b) has less plasma in the upper chromosphere (pushing the $\tau=1$ layer lower) relative to Figure 1(a). The extra chromospheric mass in Figure 1(a) could be related to dynamic mass loading from shocks or to the dense overlying magnetic field. Further analysis on the properties of the $\mathrm{h} 3$ core will be discussed using maps of the h3 intensity in various magnetic structures.

Approximately $14 \%$ of the profiles for which we have sufficient data to fit have low-quality BFM and are disregarded for statistical analysis. These points occur throughout the data set. There are several distinct types of faulty fits that are displayed in Figure 2. The most common bad fit looks similar to Figure 2(a). We have calculated the contribution to $\chi^{2}$ from three regions: far wing (window edge to h1), wing (from h1 to h2), and core (h2 to $\mathrm{h} 3$ ). The error ratio for Figure 2(a) is approximately 2:7:6 (far wing:wing:core, respectively). The wing dominates the error as the positive Gaussian profile does not accurately capture the changing slope of the profile between h1 and h2. The model attempts to split the difference so the profile is too wide at h1 and too narrow at h2. Of the $5 \times 10^{5}$ bad fits, $46 \%$ are dominated by wing errors. Figure 2(b) shows the second most common fitting problem: irregular cores. Many h3 cores tend to be flatter than the Gaussian model. This is particularly true for low- $\mu$ profiles and inside filaments. Approximately $29 \%$ of the bad fit sample is dominated by core errors.

In addition to flat cores, the double-Gaussian model is fundamentally limited in its ability to capture strong h2 asymmetries. Figure 2(c) shows one such profile, where the model fits the blue peak well, but the red peak poorly. We find that $1 \%$ of the bad fits have a good fit for one peak and very poor fit for the other. That percentage is likely higher in actuality but is difficult to statistically identify.

Some plage regions contain many bad fits. Indeed, approximately $45 \%$ of the brightest plage regions $(\max [I]>800 \mathrm{DN})$ are bad fits. Figure $2(\mathrm{~d})$ presents a typical bad fit plage profile. While the model captures the overall shape of the profile quite well, the small size of the error bars (primarily based on photon noise) magnifies the inadequacies of the double-Gaussian model. The bad plage profiles account for another $2 \%$ of the bad fits in the data set.

Figure 2(e) illustrates cosmic-ray hit data. We estimate that another $2 \%$ of bad fits are the result of anomalous spikes in the spectra. Figure 2(f) illustrates a fit where the minimization routine honed in on a single-peaked solution, where a doublepeaked solution could also work. To eliminate this category of profiles, models that are single peaked but overestimate the intensity at the line center are flagged and the minimization routine is reinitialized with an narrower positive Gaussian and deeper core. If the second BFM is still single peaked and overestimates the core intensity, the model is flagged. These profiles account for another $3 \%$ of bad fits.

\section{RESULTS}

\subsection{Variation of Profiles Based on Magnetic Structure}

Figure 3 shows the large-scale structure of the Sun in h3 intensity and the magnetic field (zooming into the electronic version of Figure 3 is suggested). The internetwork is largely faint, although there is significant small-scale structure. Bright emission surrounds magnetic concentrations, network. Plage regions are very bright in h3. We do not expect the small flux tube effects of Spruit (1976) to apply at the estimated formation height of $\mathrm{h} 3(\approx 1-3 \mathrm{Mm})$. Rather, the strong emission surrounding magnetic concentrations is related to stronger heating (Withbroe \& Noyes 1977) in the chromosphere that has been well documented in Ca II H\&K (Skumanich et al. 1975). The dimmest emission occurs in filaments. The filaments we identify in h3 are similar to those visible in BBSO $\mathrm{H} \alpha$ (Denker et al. 1999). Filaments are clear indicators of unusual temperature stratifications in the solar atmosphere $\left(T<10^{4} \mathrm{~K}\right.$ plasma at altitudes of tens of Mm; Molowny-Horas et al. 1999). The low-temperature filament is likely scattering photons out of the LOS, thus reducing the intensity in a narrow Dopplerbroadened band surrounding the rest-frame line center.

Figures 4 and 5 display two enlarged regions from Figure 3, Region 1 being quiet Sun and Region 2 being plage. We have plotted a variety of statistics in these plots that measure the components of the profiles. The emission at h1v generally originates in the upper photosphere. Based on inspection of this and additional higher spatial resolution IRIS data sets, we determine that the structures are a mix of reverse granulation and grains. At $\mathrm{h} 2 \mathrm{v}$, much of the small granular structure disappears and a diffuse halo of bright structures overlay and surround magnetic concentrations. The brightest plage is almost twice as bright as the brightest network, and the maximum $\mathrm{h} 2 \mathrm{v}$ emission occurs near the center of the magnetic flux concentration. Single-peaked profiles are relativity common $(\sim 10 \%)$ in plage, as depicted by the contiguous red regions in Figure 4. Figure 6(a) shows an example of a singlepeaked plage profile. While our algorithm separates between single-peaked and double-peaked profiles to aid in statistical analysis, there is in fact a smooth continuum of weakly separated, weakly reversed profiles that bridge those two profile categories (Figure 6(c) shows an example). In the semiempirical models of the solar atmosphere, the $\mathrm{Mg}$ II $\mathrm{h}$ source function above the temperature minimum has a local maximum at an altitude of $1.2 \mathrm{Mm}$ (see Figure 7 in Leenaarts et al. 2013a), while the line core forms near a height of $2 \mathrm{Mm}$. To produce a single-peaked profile the atmosphere must be structured such that source function does not vary significantly between $\tau=1$ at h2 and h3. At high densities, the source function closely adheres to the Planck function, which rises through the chromosphere. These profiles are consistent with the model that hot high-density loops are rooted in plage. Most sunspot profiles are single peaked, and an example is displayed in Figure 6(e). Single-peaked umbral profiles were previously reported in Lites \& Skumanich (1982) and Morrill et al. (2001). Sunspot profiles are half as bright at $\mathrm{h} 2$ and $20 \%$ narrower at $\mathrm{h} 1$ than plage. The far wing intensity (beyond h1) is lower in sunspots than plage or internetwork. Single-peaked profiles also occur in internetwork regions, albeit at much lower frequency $(\sim 1 \%)$. Figure $6(\mathrm{~b})$ shows a typical internetwork single-peaked profile. Although this profile contains a single maximum, it still requires a substantial contribution from the negative Gaussian amplitude ( $h$ in Equation (1)) to achieve a low $\chi^{2}$. Figures 6(d) and (f) show profiles that occur at low frequencies in the internetwork $(<0.0001 \%)$. These profiles represent some of the extreme variations from the mean profile shape that we observe in this large data set. The profile of Figure 6(d) could be produced by extreme upflows in the upper chromosphere that completely mask the photons in the nominal 

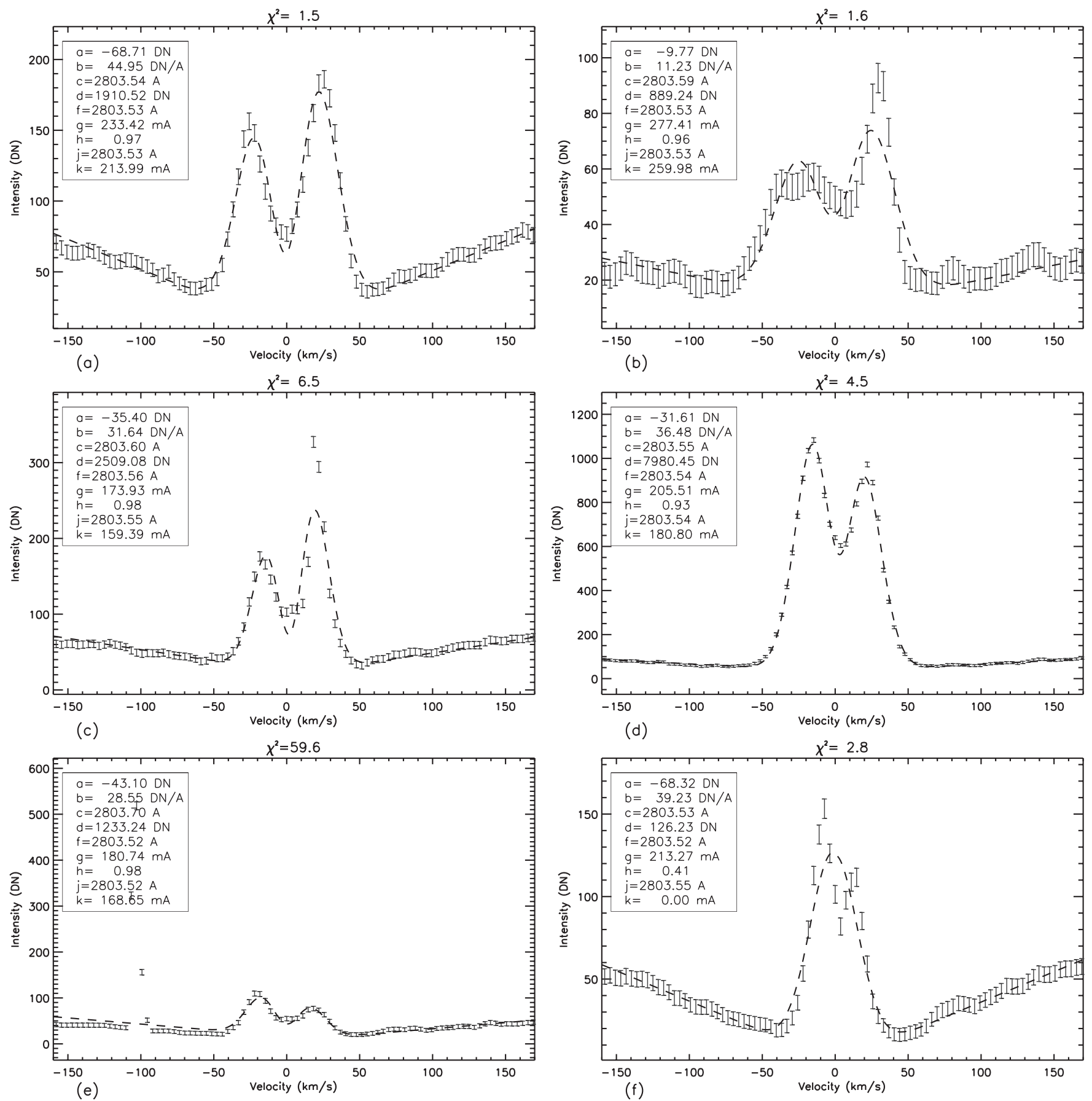

Figure 2. Profiles with high $\chi^{2}$ : non-Gaussian inner wings (a), flat core (b), non-Gaussian asymmetry (c), bright plage (d), cosmic rays (e), and faulty minimization (f).

position of the h2v peak and wing. Figure 6(f) illustrates $\mathrm{Mg}$ II $h$ in absorption, which requires that the source function monotonically decrease from the photosphere precluding the mid-chromospheric temperature rise.

The intensity structure at h3 varies significantly in the quiet Sun. As discussed in Leenaarts et al. (2013b), the Bifrost model predicts that there is a large range of formation heights for $h 3$ (see Figure 4 in that paper). Semi-empirical models also predict higher column masses at a given altitude in the enhanced network versus faint internetwork (Fontenla et al. 2009), which alters the formation height for $h 3$. The formation region of $h 3$ in the network and in the internetwork likely varies significantly depending on the magnetic topology of the upper chromosphere. Near the edge of network and extending into internetwork regions, coherent dark loop structures are visible. These are fibrils and there is a very strong contrast between the h3 intensity in and neighboring the fibril. Fibrils emanate from a fraction of network boundaries. In internetwork, it is more difficult to discern large coherent structures. It is likely that chromospheric opacity in these regions is lower than in fibrils, and we are able to observe photons that originate lower in the atmosphere. The structure at these lower heights is dominated by shocks, so we do not observe large coherent horizontal structures.

The $\mathrm{h} 2 \mathrm{v}$ intensity and the $\mathrm{h} 1 \mathrm{width}$ show similar structures, both exhibit pronounced halos around flux concentrations. If 

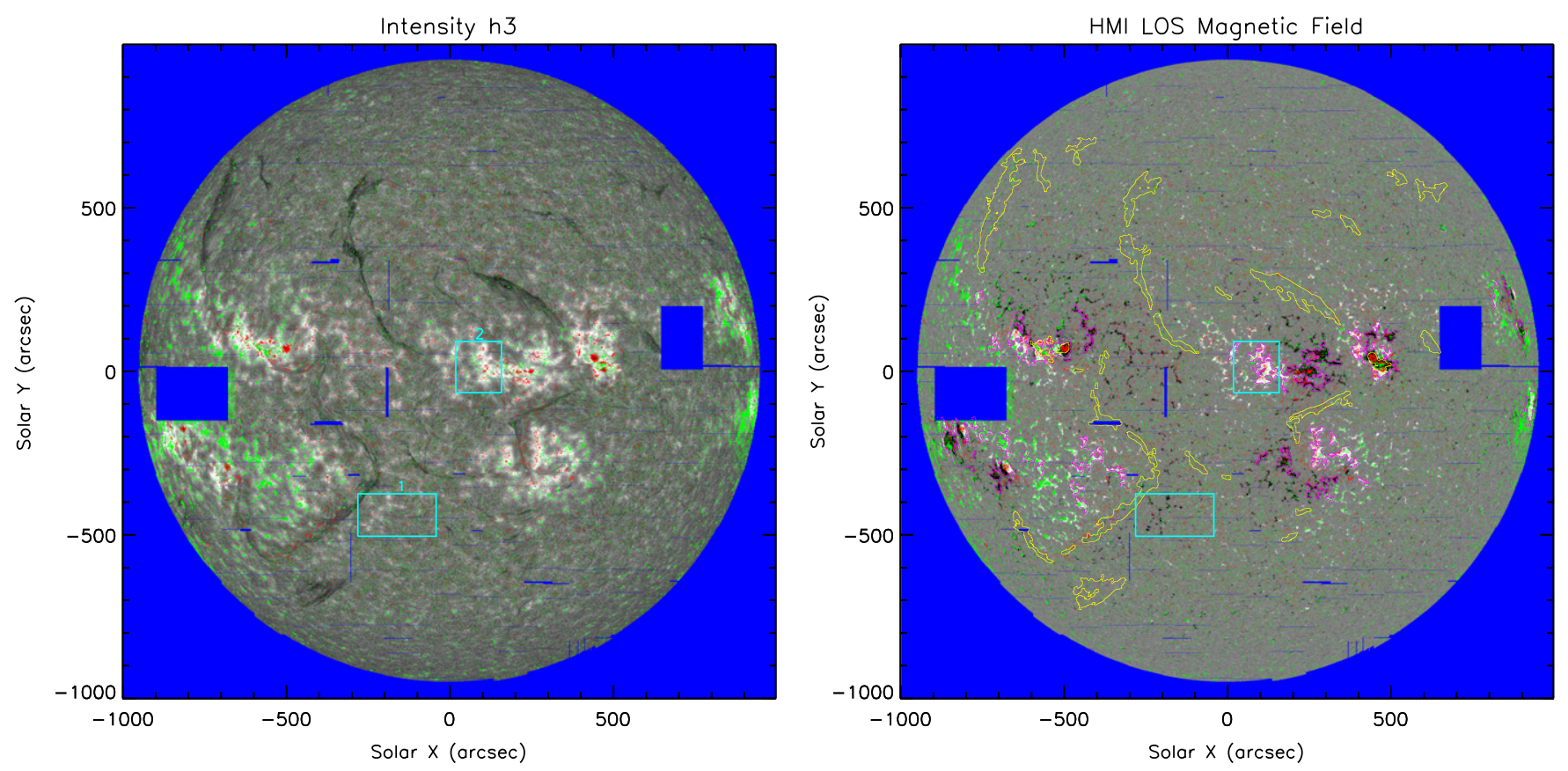

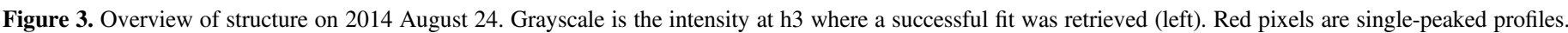

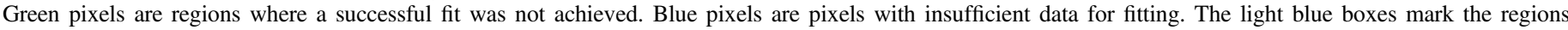
displayed in Figures 4 and 5. SDO/HMI LOS magnetic field map (right). Filaments are bordered by yellow contours, and plage is bordered by magenta.

we consider an atmosphere where the $\mathrm{Mg}$ II $\mathrm{h}$ profile can be well described by a the linear+single Gaussian model (Equation (1) with $h=0$ ), then we could expect that there would be a strong correlation between the peak intensity and the width of the profile because we are measuring the width based on the inflection point at the continuum and not the Gaussian parameter $g$ from Equation (1). In the solar example where the profiles generally are not well described as a Gaussian emission line, we would expect this effect to be reduced. In the internetwork (Solar-X between -150 and -50 and Solar-Y between -500 and -440 ), we see regions of broad $\mathrm{h} 1$ width but weak $\mathrm{h} 2 \mathrm{v}$ emission that provide a counterexample. The h2 width (also referred to as peak separation in Leenaarts et al. 2013b and Pereira et al. 2013) shows significantly more small-scale structure. The h2 width can be correlated with the range of vertical velocities present in the upper chromosphere (Leenaarts et al. 2013b). The internetwork h2 widths are not much narrower than in network or plage, despite a large difference in $I_{\mathrm{h} 2 \mathrm{v}}$. In plage and network, collimated and linear structures are visible, hinting at magnetically aligned features. The internetwork is likely traversed by a complex web of canopy fields connecting many dispersed and mixed-strength magnetic concentrations (Schrijver \& Title 2003). Flows along topologically distinct loops that cross through the LOS would not be correlated and might exhibit large velocity gradients that produce wide h2 separations. Concentrations of low h2 width can be seen surrounding single-peaked profiles in internetwork, network, and plage. As mentioned in the discussion of Figure 6, these profiles fit the gradual transition from weakly reversed double-peaked profiles to single-peaked profiles.

The Doppler shift of h3, $\lambda_{\mathrm{h} 3}$, rarely exceeds $11 \mathrm{~km} \mathrm{~s}^{-1}$. Near the edge of plage, fibrils show coherent velocity structure. In the network, it is difficult to discern any fibrils in $\lambda_{\mathrm{h} 3}$. Network actually exhibits a greater variability in h3 velocity than plage or internetwork. The h3 velocity is highly correlated with the vertical velocity at the $\tau=1$ layer. The plage flow structures are likely related to the periodic flows and transition region oscillations reported in De Pontieu et al. (2003).

\subsection{Dynamic Profile Variations}

There are profound differences in the shape of the $\mathrm{Mg}$ II $\mathrm{h}$ profile and the morphology of spatial structure based on the magnetic environment. We also know the chromosphere is buffeted by waves driven by the turbulent velocity field of the photosphere. Many wave modes impart a direct modulation of spectral profiles based on the density and velocity perturbation. In addition, these waves may add to magnetic stressing or heating, which in turn varies the thermal structure of the chromosphere on small scales. Based on these effects, we observe a high degree of variability of profiles within the magnetically classified regions.

Figure 7 displays the mixture we see of magnetic and nonmagnetic profile variability (zooming in the electronic version of Figure 7 is suggested). Each column is sorted from lowest to highest intensity at the rest wavelength line core. Overall, there is a general trend that the brightest profiles occur in a stronger magnetic field, which is consistent with the model that chromospheric heating may be induced or at least aided by magnetic fields. The brightest profiles also tend to be single peaked. The intensity tends to be higher at h2v than h2r. This is an indication that downflows predominate the chromosphere, which is well explained for shocks propagating through a nonmagnetized atmosphere in Carlsson \& Stein (1997). This effect has also previously been measured in optically thin chromospheric emission lines by Peter \& Judge (1999).

The comparison between Figures 4 and 7 is interesting. When structural information is included with the data (i.e., presented in 2D maps), it is relatively straightforward to categorize how magnetic profiles differs from non-magnetic profiles. By reducing the dimensionality of the data set (i.e., 

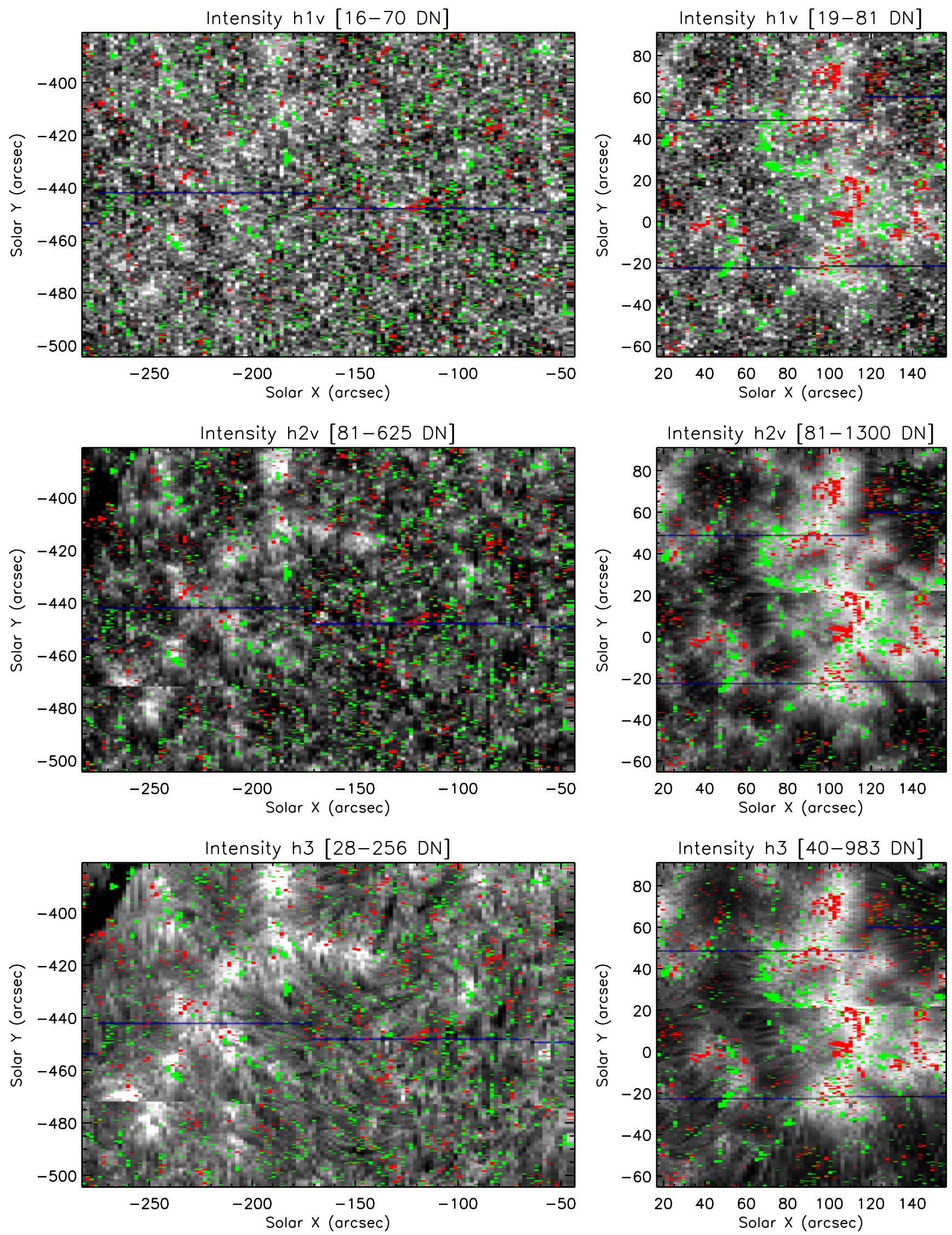

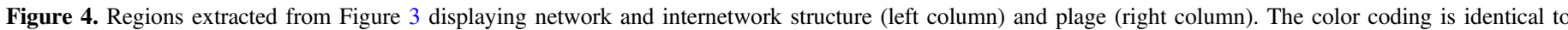
Figure 2. The color table scaling is exponential with $\gamma=0.25$. The color table bounds are listed in brackets. 
Width $\mathrm{h} 1$ [0.61-1.2 A]

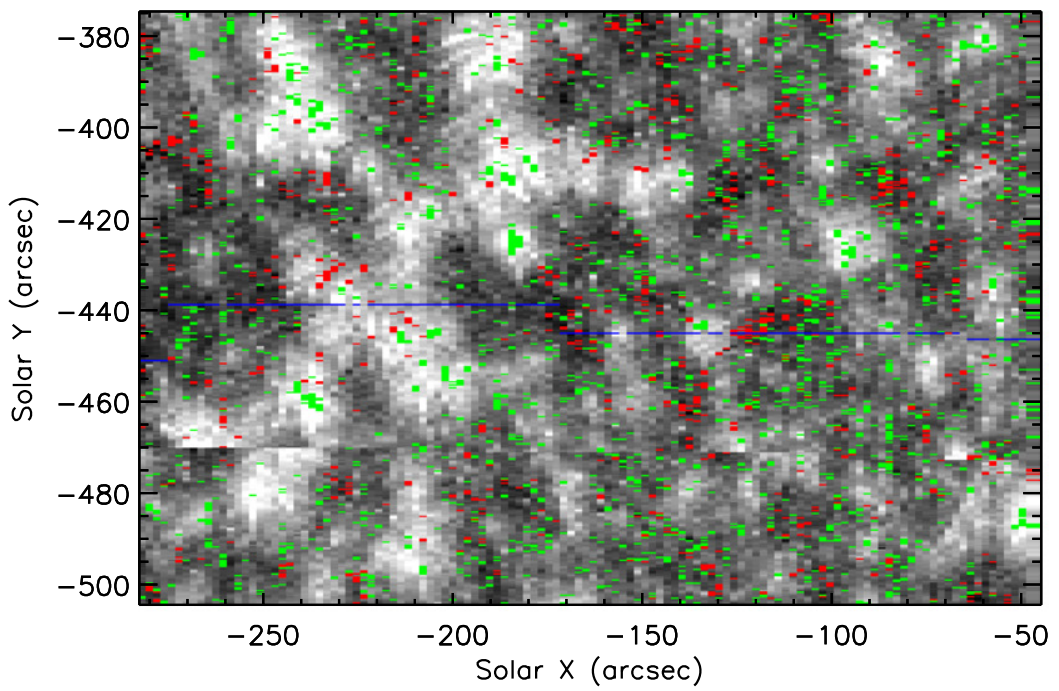

Width h2 [0.17-0.41 A]

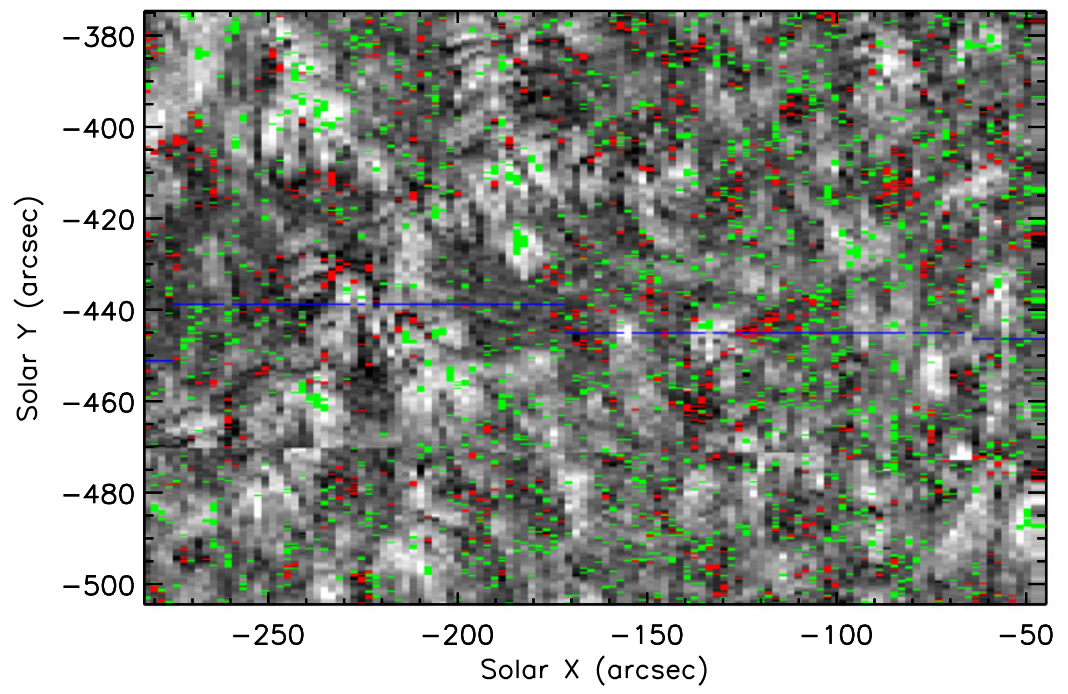

$\lambda \mathrm{h} 3\left[{ }^{\left.-11-{ }^{+} 11 \mathrm{~km} / \mathrm{s}\right]}\right.$

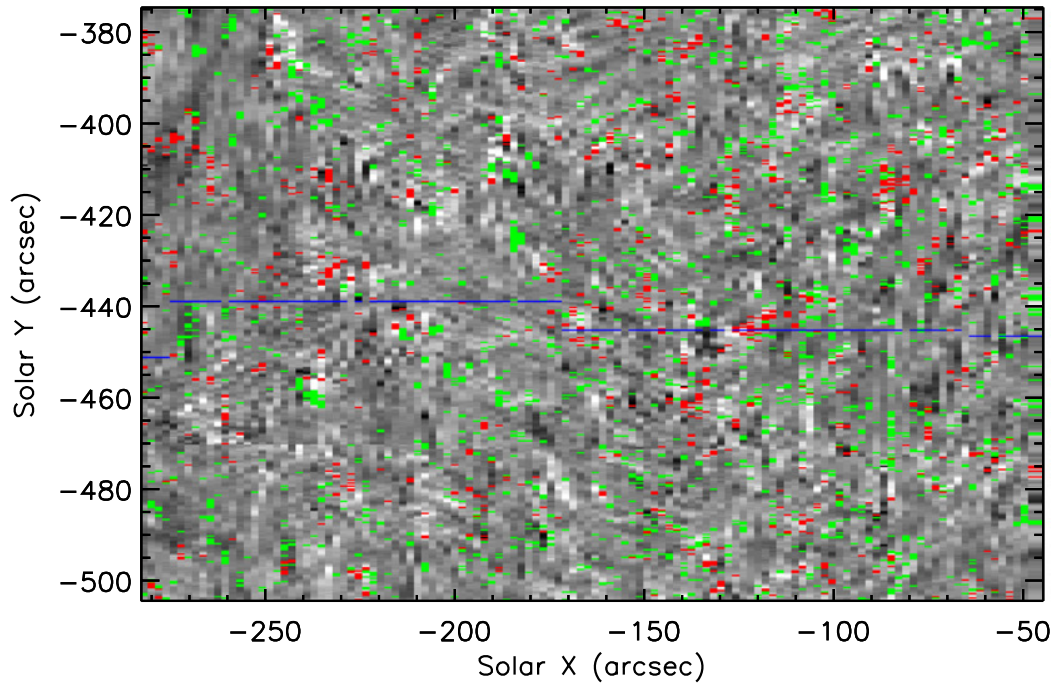

Width h1 [0.61-1.36 A]

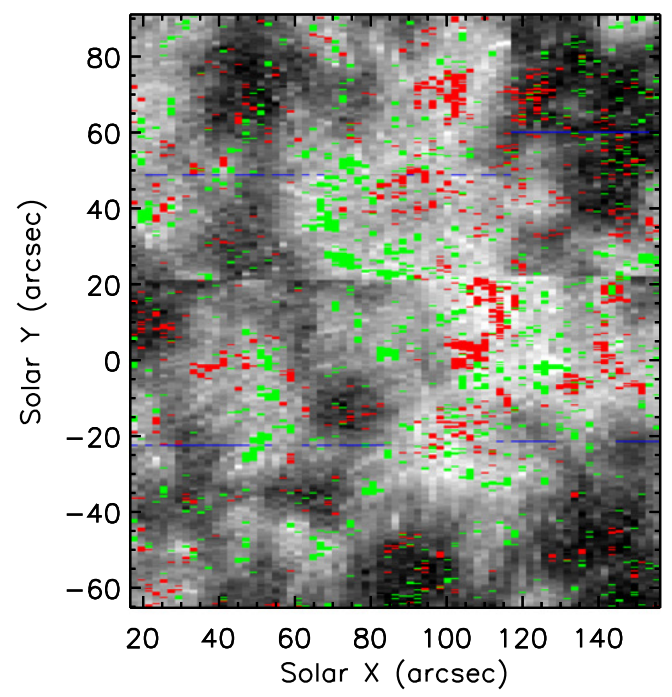

Width h2 [0.17-0.41 A]

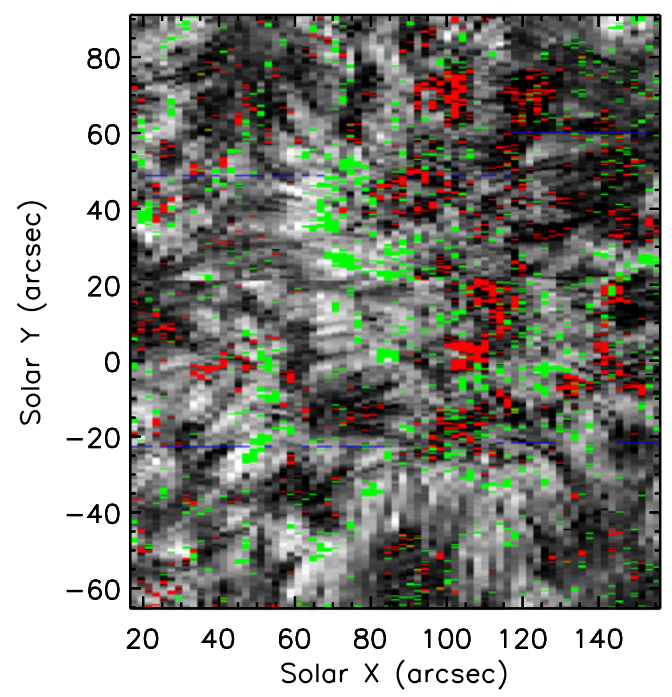

$\lambda \mathrm{h} 3\left[{ }^{\left.-11-{ }^{+} 11 \mathrm{~km} / \mathrm{s}\right]}\right.$

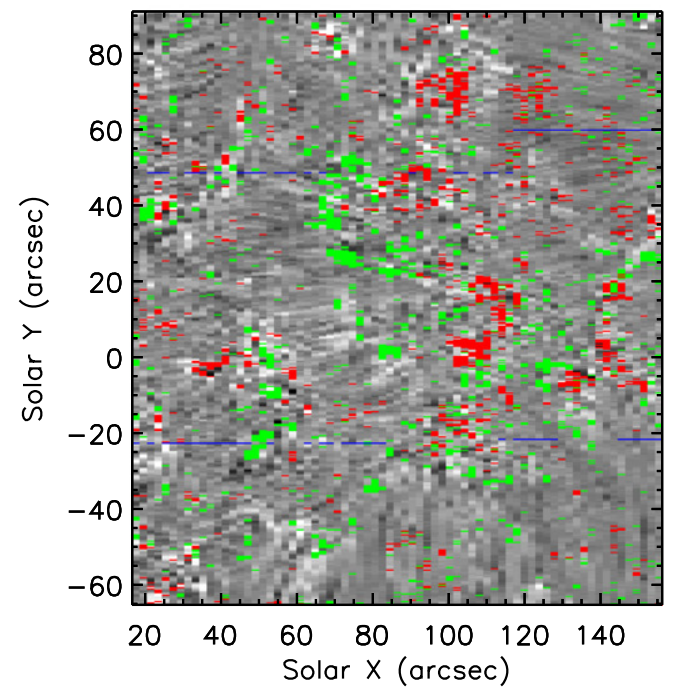

Figure 5. Same as Figure 4, but the color table scaling is linear. 

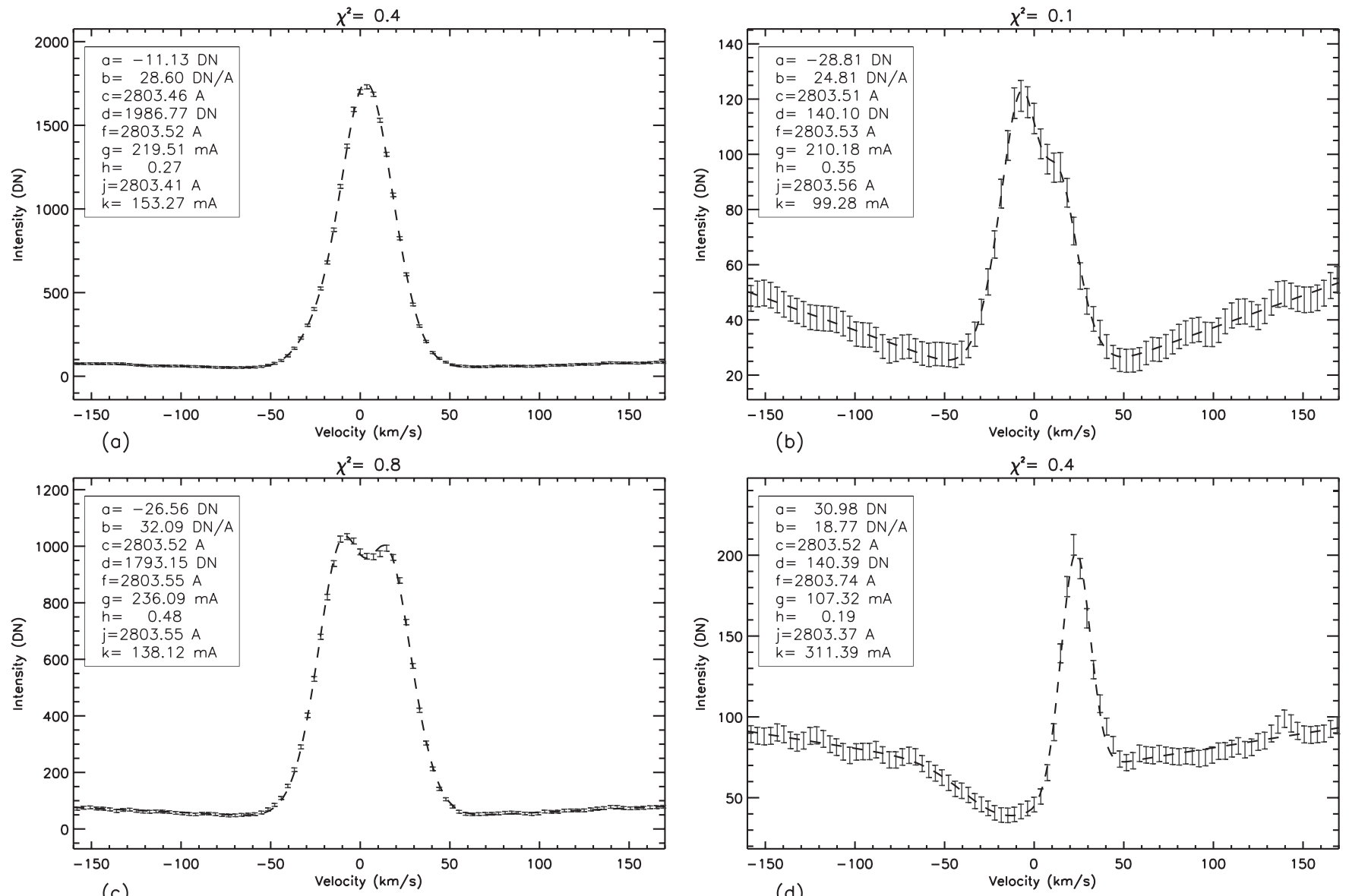

(c)
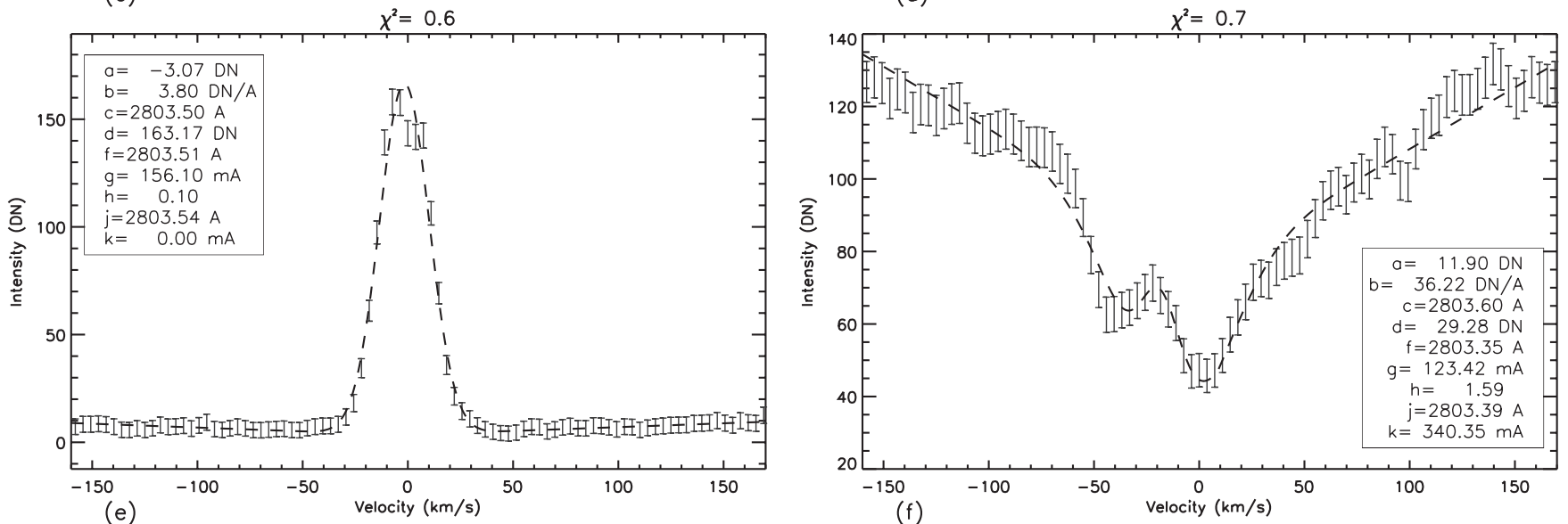

Figure 6. Single-peaked profile in plage (a), single-peaked profile in internetwork (b), weakly reversed plage profile (c), highly asymmetric single-peaked profile (d), sunspot profile (e), and reversed absorption profile (f).

presenting the profiles categorized only by magnetic field strength), the inherent variability of the profiles becomes more obvious. Nearly all the types of profiles that we observe in the internetwork also occur in strong magnetic field regions, although the fractional occurrence rate may change. Table 2 presents examples of profiles with particular characteristics that are visible in Figure 7. The examples are selected to span both the magnetic field strength as well as profile core intensity.

The variability depicted in Figure 7 is attributable to dynamics as waves and shocks modulate the intensities and shape of the profiles. These processes should have a highly patterned and repeated spectral signature, similar to the $\mathrm{H} \alpha$ sawtooth pattern attributed to passage of a shock in Hansteen et al. (2006). While our data set is ill suited for determining those patterns, the temporal signature of the dynamics should be engrained in the distribution of the profiles. In Section 4.3, we present measurements on the mean and variance of a number of profile statistics. In addition to averaging the effect of dynamics into a single archetypal profile, it also important to consider the coordinated effects these dynamics have on the shape of the profile. To extract these relationships from our data set, we calculate a cross correlation matrix of the profile components, presented in Table 3. The correlation coefficients allow us to measure if the variability of a particular statistic 


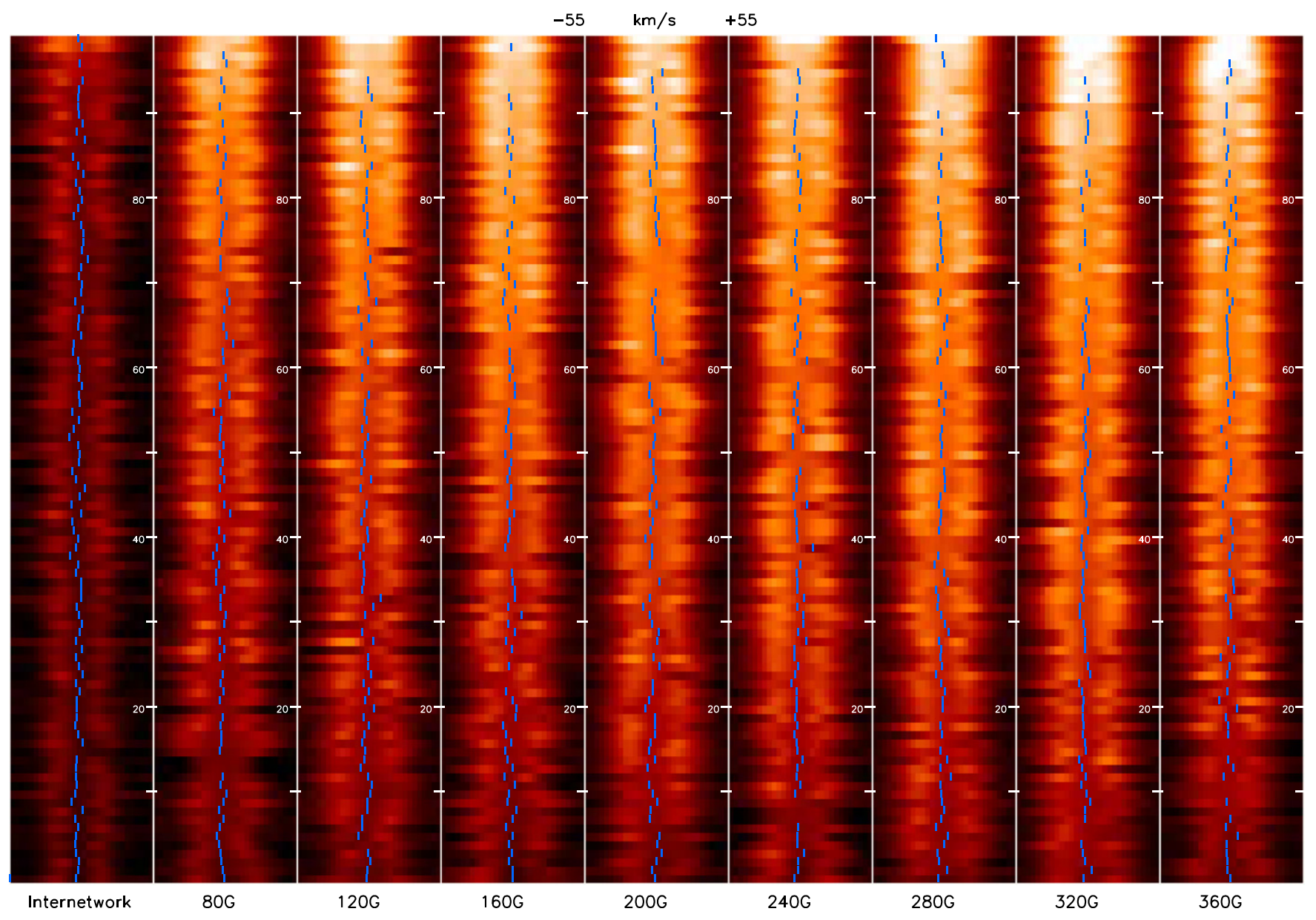

Figure 7. Example low- $\chi^{2}$ profiles from near disk center. On the $y$-axis are 100 stacked profiles sorted by the intensity at $2803.5 \AA\left(v=0 \mathrm{~km} \mathrm{~s}{ }^{-1}\right)$. Each $\mathrm{x}$-column represents profiles from regions defined by HMI LOS field strength. The spectral width of each column is $\pm 55 \mathrm{~km} \mathrm{~s}^{-1}$. The color table is exponential with $\gamma=0.25$ and represents the spectral intensity. Each profile with a well-defined h3 minimum has it marked with a blue bar.

Table 2

Notable Profiles in Figure 7

\begin{tabular}{lc}
\hline \hline Profile Characteristic & Examples (Column and Rank) \\
\hline Unshifted Single Peak & IN-86, 120G-99, 360G-8, 360G-99 \\
Shifted Single Peak & IN-15, 360G-30 \\
Wide h1 & IN-28, IN-95, 200G-33, 320G-89 \\
Narrow h1 & IN-3, 240G-19, 240G-82 \\
Wide h2 & IN-6, 200G-63, 240G-65 \\
Narrow h2 & 80G-28, 80G-97, 160G-25, 360G-96 \\
Asymmetric $\left(I_{\mathrm{h} 2 \mathrm{v}}>I_{\mathrm{h} 2 \mathrm{r}}\right)$ & IN-43, 120G-28, 200G-86, 360G-75 \\
Asymmetric $\left(I_{\mathrm{h} 2 \mathrm{r}}<I_{h 3 r}\right)$ & $80 \mathrm{G}-55,320 \mathrm{G}-14,320 \mathrm{G}-72$ \\
Shifted h3 & IN-73, 80G-55, 120G-34, 360G-44, 360G-77
\end{tabular}

Note. Coordinates label the magnetic field and rank.

occurs synchronously with any other statistic. Here, the cross correlation of statistics $S_{a}$ and $S_{b}$ is defined as

$$
C_{a b}=\sum_{i j} \frac{\left(S_{a}^{i j}-\overline{S_{a}}\right)\left(S_{b}^{i j}-\overline{S_{b}}\right)}{\sigma_{a} \sigma_{b}},
$$

where $S_{n}^{x y}$ represents statistic $n$ at spatial position $x y, \overline{S_{n}}$ is the mean, and $\sigma_{n}$ is the variance of statistic $n$. $C_{a b}$ varies between -1 (anti-correlation) and 1 (correlation). We have limited our profile subset to high-quality double-peaked fits of near disk center internetwork so as to limit the scope of variability to fine
Table 3

Cross Correlation Coefficients for Components of Internetwork Profiles

\begin{tabular}{|c|c|c|c|c|c|c|c|}
\hline & Int. h1v & Int. h2v & Depth h3 & Asym. h2 & Width h1 & Width h2 & Vel. h3 \\
\hline Int. h1v & 1.00 & 0.33 & 0.11 & 0.05 & 0.22 & 0.31 & 0.06 \\
\hline Int. h2v & 0.33 & 1.00 & 0.18 & 0.43 & 0.41 & 0.10 & 0.36 \\
\hline Depth h3 & 0.11 & 0.18 & 1.00 & 0.18 & -0.10 & 0.46 & 0.00 \\
\hline Asym. h2 & 0.05 & 0.43 & 0.18 & 1.00 & 0.00 & 0.01 & 0.80 \\
\hline Width h1 & 0.22 & 0.41 & -0.10 & 0.00 & 1.00 & 0.64 & 0.04 \\
\hline Width h2 & 0.31 & 0.10 & 0.46 & 0.01 & 0.64 & 1.00 & -0.03 \\
\hline Vel. h3 & 0.06 & 0.36 & 0.00 & 0.80 & 0.04 & -0.03 & 1.00 \\
\hline
\end{tabular}

Note. The ensemble of profiles only uses models with $\chi^{2}<1.5$ and $\mu>0.75$. Abbreviations: Int., intensity; Asym., asymmetry; Vel., velocity.

structure and dynamics and not viewing angle or magnetic structure. The variables that are most well correlated are the h3 wavelength and and h2 asymmetry. Because the far wings of the profile extend deeper into the atmosphere and do not shift in wavelength dramatically due to the low sound speed, this correlation is expected based on our double-Gaussian model. The more misaligned the negative Gaussian is from the positive Gaussian, the larger the $\mathrm{h} 2$ asymmetry. Given the success rate of our fitting algorithm and the quality of the fits, we believe 
this effect is robust against the bias of the fit model. The $\mathrm{h} 1$ width and the h2 width are also strongly correlated. Based on a scaling law argument, Ayres (1979) suggests that the ratio of $\mathrm{h} 1$ and $\mathrm{h} 2$ is determined by the vertical extent of the chromosphere and rate of chromospheric heating. We expect that deeper profiles (high $D_{\mathrm{h}}$ ) would produce wider h2 separation and thus have a strong correlation. The relatively high correlation between h2 asymmetry and h2v intensity is an artifact. By comparing asymmetry with h2r intensity and h-line radiance, we find brighter profiles are generally more symmetric. The preponderance of positive asymmetry profiles biases the sample.

These correlations are best used in conjunction with rapid cadence raster scans that allow us to extract both spatial and temporal information on the evolution of profiles across wellresolved spatial structures. In particular, we need to further develop studies that link the variable chromospheric profiles with the underlying photospheric drivers.

\subsection{Center-to-limb Variations}

The viewing angle changes the observed intensities and shape of the profile because the column densities and velocity projections are dependent on the line of sight. Figures 8 and 9 illustrate how the viewing angle affects the profiles in our three well-populated magnetic subsets using joint probability distribution plots. The internetwork is by far the largest subset and is evenly distributed in $\mu$. The center-to-limb trends (slopes) are negative in $\mathrm{h} 1, \mathrm{~h} 2$, and $\mathrm{h} 3$ intensity (similar to optical continuum) and positive in h1 and h2 widths. The h2 and h3 intensity distribution are more dispersed at center than limb. This may be revealing that atmospheric thermal anisotropies decrease as a function of altitude. As constructed, there is no way to account for the spread of these statistics (at a given angle) using the semi-empirical models. The asymmetry and depth statistics are unaffected by varying $\mu$.

Given the clustered distribution of magnetic flux, the distribution of plage and network fields are less uniform statistical samples. The magnetic field strength in plage varies significantly from region to region and plays a role in the scatter of the $I_{\mathrm{h} 2 \mathrm{v}}$ and $I_{\mathrm{h} 3}$ distributions. Plage and network are brighter than internetwork and wider at h1. For nearly all statistics, the distributions are more dispersed than internetwork. The h1 widths are well correlated with viewing angle. The h2 widths have a wider spread with an extended low-width tail. Plage and network are less asymmetric and have shallower cores compared to the internetwork. In order to measure radiance in the line, we have summed the data between $\mathrm{h} 1 \mathrm{v}$ and $\mathrm{h} 1 \mathrm{r}$. In the radiance near disk center, network is a factor of three brighter than internetwork, while plage is a factor of eight brighter. Network is more dispersed in h3 velocity than internetwork or plage. Given the strong flows often observed in active region coronal spectra, it is surprising that network exhibits a higher percentage of highvelocity profiles than plage.

Avrett et al. (2013) discussed the center-to-limb variation of the $\mathrm{Mg}$ II $\mathrm{k}$ profiles based on the predictions of a series of semiempirical models of the solar atmosphere. In that paper, Model B represents the mean internetwork, while Model F represents the bright network. Table 4 compares the spectral intensities of the model and our derived profile statistics. While the semiempirical models capture the variation of the emission peak (between internetwork and network and center-to-limb), it does not capture the variation in the core. The semi-empirical model predicts relatively little variation in the core intensity as a function of $\mu$, while we observe a measurable decline in the core at the limb. As discussed in Section 4.1, if the formation height of h3 is highly variable then the limb-darkening effect can be explained by the increased sampling of high-lying and dark fibrils at low $\mu$.

The semi-empirical models are constructed as a tool to incorporate a broad range of spectral information and convert it into a consistent model of the atmosphere. These static models provide us information on the structure of an averaged solar atmosphere, while the 3D MHD models provide information on the dynamics based on physics but limited by the computational power. By comparing the IRIS data with these models, we increase our understanding of this complex data set and identify portions of the model that require adaptation.

\section{CONCLUSIONS}

Most of our understanding of the chromosphere is based on ground-based observations of $\mathrm{Ca}$ II and $\mathrm{H} \alpha$. While data sets like that of Swedish Solar Telescope/CRISP provide us with the highest spatial resolution measurements of the chromosphere, they are limited in spatial FOV and the often brief periods of prime atmospheric seeing. The IRIS spacecraft has opened up a new window into the chromosphere, with the ability to resolve sub-arcsec structure and the stability and atmosphere-free seeing to produce long-duration data sets. One of the primary purposes of the analysis of solar spectral lines is to diagnose the physical processes and the thermodynamic conditions of the atmosphere. Prior to establishing diagnostics, it is essential to identify the methodology for measuring the necessary spectral features, and our works fits into this first step. The $\mathrm{Mg}$ II $\mathrm{k}$ line differs slightly from $\mathrm{Mg}$ II $\mathrm{h}$ in formation height due to the difference in oscillator strength. It is important to extend this analysis to that line for additional diagnostic capabilities; however, our method requires adaptation. The Mn I line at 2795.6 $\AA$ can blend with the $\mathrm{k}$ line for wide profiles. A wellconstrained fit model can likely be constructed with a spectral window that also includes the unblended Mn I 2999.1 A line. This is a likely direction for future analysis.

Leenaarts et al. (2013a, 2013b) and Pereira et al. (2013) have used an advanced numerical simulation of the solar atmosphere to forward model $\mathrm{Mg}$ II h\&k emission and compare the derived spectral information with the physical properties of the emitting plasma. Many of the statistics we have measured in this data set are identical to those discussed by those authors. A direct comparison between the modeled profiles and observed profiles is difficult. While numerical models have proven powerful in describing specific physical processes (the formation of shocks by high-frequency vertical velocity fluctuations described the Carlsson \& Stein 1997, for example), the models are not producing many of the spectral features we identify in the observations. We know that the observed profiles are significantly broader than those forward modeled by Bifrost. Although the forward model incorporates magnetic fields, the limited scale and largely bipolar distribution of magnetic elements are not direct analogs to all solar structures. As reported in Leenaarts et al. (2012), the 3D radiation field (as opposed to a 1D vertical flux) can have a strong effect on the observed structure of the chromosphere. Ultimately, the distribution of $\mathrm{Mg}$ II ions, the relative populations of the ground and doublet states, and the incident radiation field along 

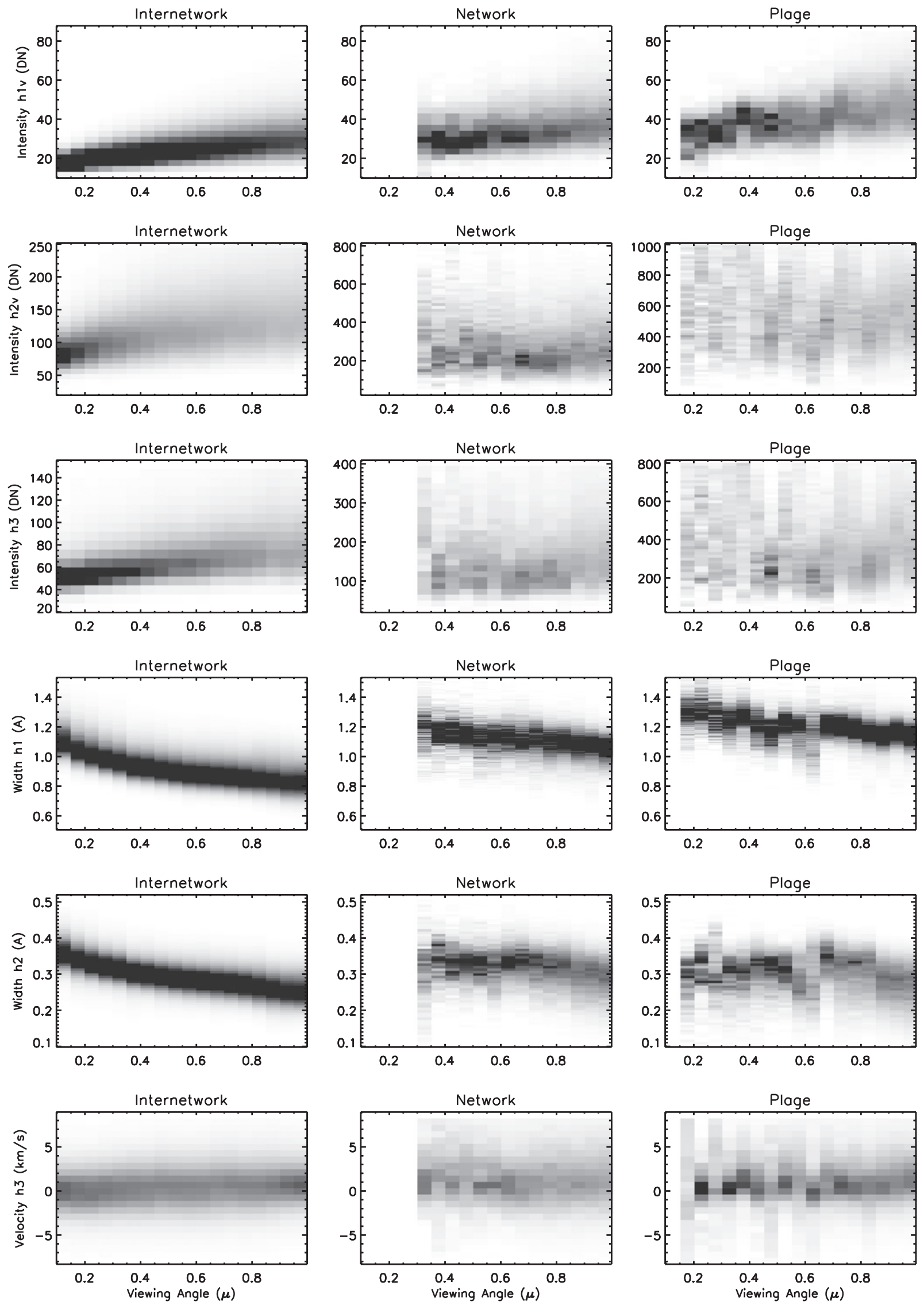

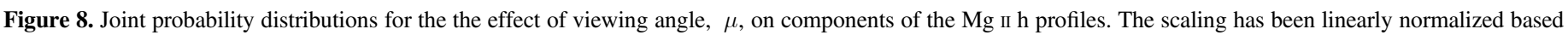
on bin size and number of applicable profiles at each viewing angle. The maximum occurrence rate is uniform across structures but varies per statistic. 

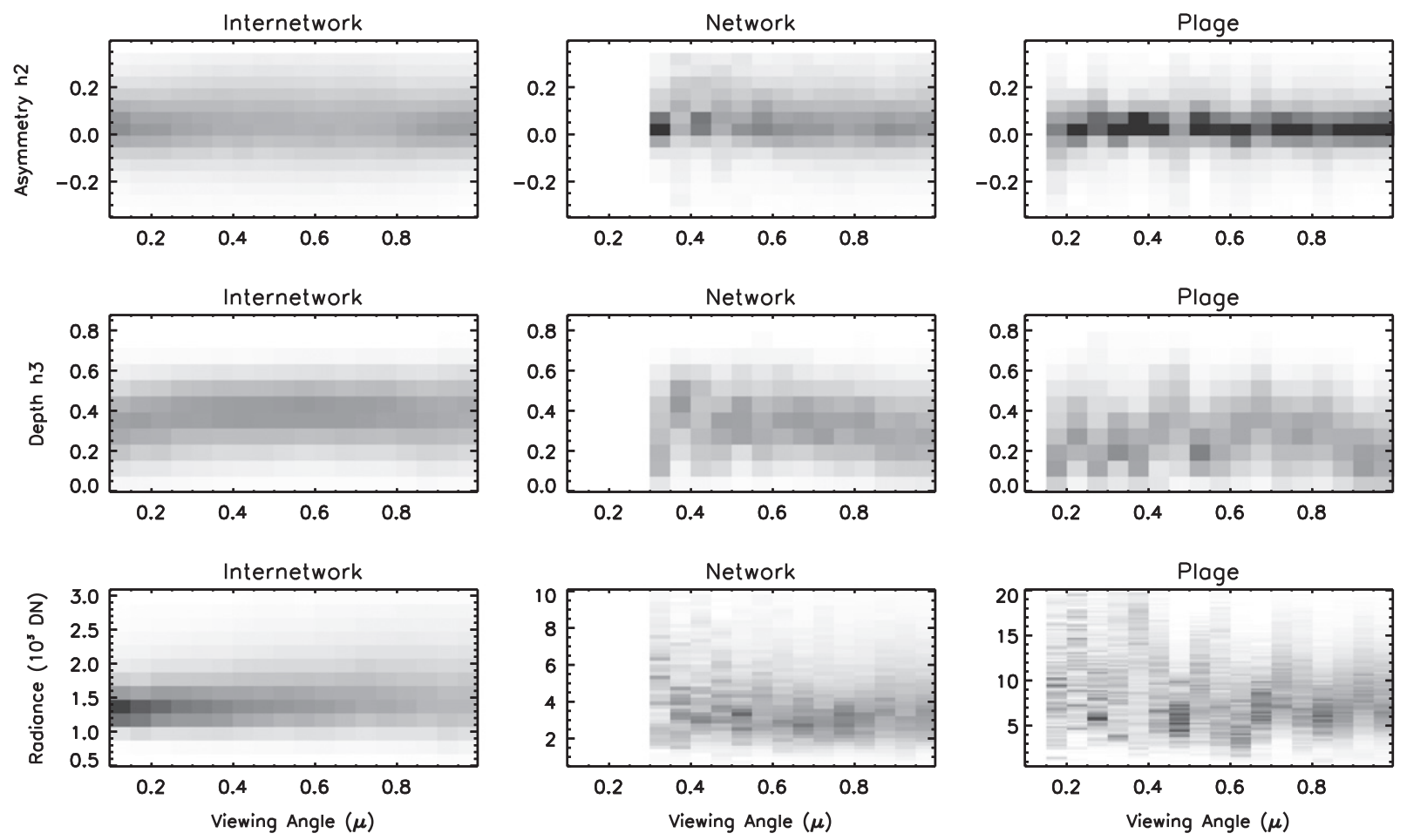

Figure 9. Same as Figure 8.

Table 4

Comparison of Center-to-limb Variation from Avrett et al. (2013, ALM) for Mg II k and the Measured Quantities with IRIS for Mg II h in Internetwork (IN and Model B) and Network (NW and Model F)

\begin{tabular}{|c|c|c|c|c|}
\hline Statistic & ALM $^{\mathrm{a}}$ Model B & ALM $^{\mathrm{a}}$ Model F & IRIS $^{b}$ IN & $\overline{\text { IRIS }^{\mathrm{b}} \mathrm{NW}}$ \\
\hline Peak Intensity, $\mu=1.0$ & 210 & 390 & 170 & 280 \\
\hline Peak Intensity, $\mu=0.1$ & 140 & 270 & 90 & $\cdots$ \\
\hline Core Intensity, $\mu=1.0$ & 40 & 130 & 90 & 200 \\
\hline Core Intensity, $\mu=0.1$ & 40 & 120 & 50 & $\cdots$ \\
\hline Depth, $\mu=1.0$ & 0.81 & 0.67 & 0.47 & 0.29 \\
\hline Depth, $\mu=0.1$ & 0.71 & 0.56 & 0.44 & $\ldots$ \\
\hline
\end{tabular}

Notes.

${ }^{a}$ ALM intensity units are $\mathrm{W} \mathrm{m}^{-2} \mathrm{sr}^{-1} \AA^{-1}$.

${ }^{\mathrm{b}}$ IRIS intensity units of DN.

the line of sight are the determining factors for the shape of the emergent $\mathrm{Mg}$ II $\mathrm{h}$ profile. Both semi-empirical and MHD atmospheres provide us a means of calculating that distribution with strict limits on which physics can be included.

The analysis of optically thick spectral lines present researchers with a double-edged sword: the shape of the emergent profile encodes the thermal and hydrodynamic information of a significant vertical swath of the atmosphere, but the physical quantities we ultimately seek to measure are entangled. One potential diagnostic is a Mg II profile inversion, similar to those used for optical spectropolarimetric data (Ruiz Cobo \& del Toro Iniesta 1992). Of course inversions codes have largely operated in the regime where LTE is a reasonable approximation, which is not true for $\mathrm{Mg}$ II. The next step in this research is the integration of our derived quantities into our physical models (cartoon or otherwise) of the how the dynamic chromosphere is structured.

In this paper, we have dissected a data set containing $\mathrm{Mg}$ II $\mathrm{h}$ spectral profiles over the full solar disk. We decomposed the data set into structural regions based on the magnetic field and quantified the differences between the regions based on a number of profile statistics. The internetwork is dim at h2 and deeply reversed. The network is brighter at $\mathrm{h} 1, \mathrm{~h} 2$, and $\mathrm{h} 3$ than internetwork. It has enhanced h1 width compared with internetwork. Plage is brighter and more variable at h2 than network or internetwork. It has a small h2 width and weak reversal. Single-peaked profiles occur all over the disk, but most commonly in plage. We have created maps of many statistics for the line profiles. There are coherent but varied structures visible in width, intensity, and velocity statistics. Fibrils are primarily visible in plage in $\lambda_{\mathrm{h} 3}$ and in network in $I_{\mathrm{h} 3}$. Both strong and weak field regions exhibit a great deal of profile variability that is the result of chromospheric dynamics. We find strong correlations for the Doppler shift of the h3 core and the asymmetry in the $\mathrm{h} 2$ peaks and also between the widths of $\mathrm{h} 1$ and $\mathrm{h} 2$. We calculated the center-to-limb variation of a number of statistics and compare them with the predictions of a semi-empirical model. We find that both h3 and h2 exhibit limb darkening such that the core depth is flat as a function of $\mu$, while the semi-empirical model does not predict a strong limb 
darkening for $\mathrm{h} 3$. Our measurement of this effect is most robust in internetwork. This study presents the most comprehensive observational study on solar $\mathrm{Mg}$ II spectra to date, but it is clear that there are many facets on the formation and variability of this spectral line that are still not fully understood. It is essential to keep developing atmospheric models that can accommodate the great deal of dynamical and structural variability we observe in high-resolution observations of the chromosphere. The photospheric velocity field strongly modulates the plasma conditions and radiation field in the lower chromosphere, and the upper chromosphere is highly structured by the magnetic field in the $\beta \leqslant 1$ regime. Both of these boundary conditions must be considered in our future modeling efforts.

IRIS is a NASA small explorer mission developed and operated by LMSAL with mission operations executed at NASA Ames Research Center and major contributions to downlink communications funded by ESA and the Norwegian Space Center.

\section{REFERENCES}

Artzner, G., Bonnet, R. M., Vial, J. C., et al. 1977, SSI, 3, 131

Avrett, E., Landi, E., \& McKillop, S. 2013, ApJ, 779, 155

Ayres, T. R. 1979, ApJ, 228, 509

Basri, G. S., \& Linsky, J. L. 1979, ApJ, 234, 1023

Blanco, C., Bruca, L., Catalano, S., \& Marilli, E. 1982, A\&A, 115, 280

Bonnet, R. M. 1981, SSRv, 29, 131

Carlsson, M., \& Stein, R. F. 1997, ApJ, 481, 500

De Pontieu, B., Erdélyi, R., \& de Wijn, A. G. 2003, ApJL, 595, L63

De Pontieu, B., Title, A. M., Lemen, J. R., et al. 2014, SoPh, 289, 2733

Dempsey, R. C., Neff, J. E., Thorpe, M. J., et al. 1996, ApJ, 470, 1172

Denker, C., Johannesson, A., Marquette, W., et al. 1999, SoPh, 184, 87

Doschek, G. A., \& Feldman, U. 1977, ApJS, 35, 471

Durand, E., Oberly, J. J., \& Tousey, R. 1949, ApJ, 109, 1

Fontenla, J. M., Curdt, W., Haberreiter, M., Harder, J., \& Tian, H. 2009, ApJ, 707,482

Gouttebroze, P. 1977, A\&A, 54, 203
Gouttebroze, P. 1989, ApJ, 337, 536

Gouttebroze, P., \& Lemaire, P. 1974, A\&A, 34, 375

Gudiksen, B. V., Carlsson, M., Hansteen, V. H., et al. 2011, A\&A, 531, A154

Guo, J., Wan, W., Forbes, J. M., et al. 2007, JGRA, 112, 10308

Gurman, J. B. 1984, SoPh, 90, 13

Hansteen, V. H., De Pontieu, B., Rouppe van der Voort, L., van Noort, M., \& Carlsson, M. 2006, ApJL, 647, L73

Henze, W., \& Stenflo, J. O. 1987, SoPh, 111, 243

Kneer, F., Mattig, W., Scharmer, G., et al. 1981, SoPh, 69, 289

Lean, J. L., Picone, J. M., \& Emmert, J. T. 2009, JGRA, 114, 7301

Leenaarts, J., Carlsson, M., \& Rouppe van der Voort, L. 2012, ApJ, 749, 136

Leenaarts, J., Pereira, T. M. D., Carlsson, M., Uitenbroek, H., \& De Pontieu, B. 2013a, ApJ, 772, 89

Leenaarts, J., Pereira, T. M. D., Carlsson, M., Uitenbroek, H., \& De Pontieu, B. 2013b, ApJ, 772, 90

Lemaire, P., Gouttebroze, P., Vial, J. C., \& Artzner, G. E. 1981, A\&A 103,160

Lemaire, P., \& Skumanich, A. 1973, A\&A, 22, 61

Linsky, J. L., \& Haisch, B. M. 1979, ApJL, 229, L27

Lites, B. W., \& Skumanich, A. 1982, ApJS, 49, 293

Markwardt, C. B. 2009, in ASP Conf. Ser. 411, Astronomical Data Analysis Software and Systems XVIII, ed. D. A. Bohlender, D. Durand \& P. Dowler (San Francisco, CA: ASP), 251

Milkey, R. W., \& Mihalas, D. 1974, ApJ, 192, 769

Molowny-Horas, R., Heinzel, P., Mein, P., \& Mein, N. 1999, A\&A, 345, 618

Morrill, J. S., Dere, K. P., \& Korendyke, C. M. 2001, ApJ, 557, 854

Morrill, J. S., \& Korendyke, C. M. 2008, ApJ, 687, 646

Pereira, T. M. D., Leenaarts, J., De Pontieu, B., Carlsson, M., \& Uitenbroek, H. 2013, ApJ, 778, 143

Pesnell, W. D., Thompson, B. J., \& Chamberlin, P. C. 2012, SoPh, 275, 3

Peter, H., \& Judge, P. G. 1999, ApJ, 522, 1148

Ruiz Cobo, B., \& del Toro Iniesta, J. C. 1992, ApJ, 398, 375

Scherrer, P. H., Schou, J., Bush, R. I., et al. 2012, SoPh, 275, 207

Schrijver, C. J., \& Title, A. M. 2003, ApJL, 597, L165

Skumanich, A., Smythe, C., \& Frazier, E. N. 1975, ApJ, 200, 747

Solomon, S. C., \& Qian, L. 2005, JGRA, 110, 10306

Spruit, H. C. 1976, SoPh, 50, 269

Staath, E., \& Lemaire, P. 1995, A\&A, 295, 517

Uitenbroek, H., Dupree, A. K., \& Gilliland, R. L. 1998, AJ, 116, 2501

Vial, J. C., Gouttebroze, P., Artzner, G., \& Lemaire, P. 1979, SoPh, 61, 39

Withbroe, G. L., \& Noyes, R. W. 1977, ARA\&A, 15, 363 